DOI:

10.1038/nrg2152

URLs

\title{
Onwards and upwards for genome-wide association studies
}

A consortium of 50 UK-based research groups has published its findings from a joint genome-wide association (GWA) study for seven common diseases. Using data from large numbers of individuals with these conditions, this study both identifies new genetic associations and provides important insights for the design of GWA studies.

The Wellcome Trust Case Control Consortium genotyped 2,000 affected white European UK residents for each of bipolar disorder, coronary artery disease, Crohn's disease, hypertension, rheumatoid arthritis and types 1 and 2 diabetes. Instead of picking controls for each disease, the study used a joint control group of 3,000 people. Samples from cases and controls were genotyped using the Affymetrix 500K Mapping Array set, which is designed to cover common SNPs from across the genome.

Supporting the validity of its approach, the Consortium found many associations that have been replicated in previous studies. Several new and highly significant associations also came out of the study, most of which have since been replicated, and many less strongly supported associations remain to be followed up. As well as providing new directions for investigating the genetic basis of susceptibility to the conditions that were investigated, the results provide information about the genetic architecture of common diseases. The variants that were uncovered are expected to make modest contributions to disease susceptibility, in keeping with suggestions that variants that have large phenotypic effects are unlikely to contribute to such conditions.

This study also allowed several uncertainties about the design of GWA studies to be explored. For example, the idea of using a shared control group that hasn't been specifically phenotyped for comparison with each individual disease has raised concerns. However, the strength of the results in this study suggest that this is not a major problem. Another issue is underlying population structure, which could confound results if differing ancestry among cases and controls equates to differences in the likelihood of developing a disease. However, this study found that the effects of such population stratification is modest across most of the genome, at least for the UK population.

The Consortium has made its data, results and software freely available (see links from the web site listed below), providing an important resource for other researchers who are investigating the genetic basis of susceptibility to complex diseases.

Louisa Flintoft

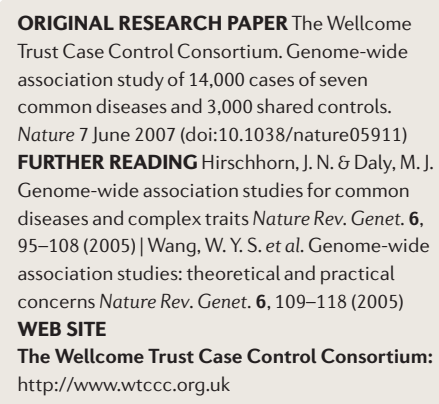

ORIGINAL RESEARCH PAPER The Wellcome Trust Case Control Consortium. Genome-wide association study of 14,000 cases of seven common diseases and 3,000 shared controls. Nature 7 June 2007 (doi:10.1038/nature05911) FURTHER READING Hirschhorn, J. N. \& Daly, M. . Genome-wide association studies for common diseases and complex traits Nature Rev. Genet. 6, 95-108 (2005) | Wang, W. Y. S. et al. Genome-wide association studies: theoretical and practical concerns Nature Rev. Genet. 6, 109-118 (2005) WEB SITE

The Wellcome Trust Case Control Consortium: http://www.wtccc.org.uk

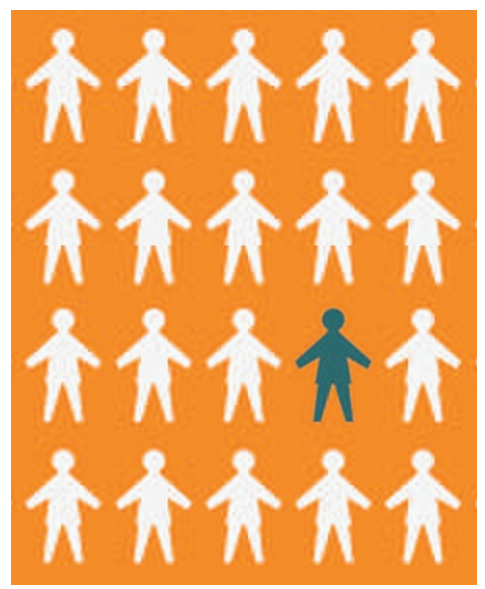

